\title{
Linear growth failure induced by systemic inflammation inhibiting IGF-1/IGFBP axis in rats with asymptomatic colitis
}

\author{
Xiaoyang Sheng ${ }^{1}$, Xueqing Sun ${ }^{2}$, Feng $\mathrm{Li}^{1}$, Junli Wang ${ }^{1}$ and Jingqiu $\mathrm{Ma}^{3^{*}}$
}

\begin{abstract}
Background: Children in poor areas show significant growth retardation that does not improve with an adequate supply of energy and nutrients, which may be related to asymptomatic intestinal infection caused by poor sanitation. Our objective was to explore the mechanism of intestinal inflammation inhibiting growth in the setting of asymptomatic colitis.
\end{abstract}

Methods: Forty-eight 3-week-old Wistar rats were randomly divided into three groups: the control group, colitis group (with asymptomatic colitis induced by $2.5 \%$ trinitrobenzenesulphonic acid) and pair-fed group (daily food intake matched to the pair in the colitis group). The linear growth was assessed, and the plasma levels of hormone and systemic cytokines were detected and compared by independent two-sample t-test or one-way ANOVA among groups.

Results: At d5, the increases in the body length of the control, colitis and pair-fed groups were $1.65 \pm 0.34 \mathrm{~cm}$, $1.10 \pm 0.30 \mathrm{~cm}$ and $1.38 \pm 0.26 \mathrm{~cm}$, respectively, and the increase in the body length in the colitis group was significantly less than that in the control group $(P<0.05)$. There were significant differences in the levels of hormone and cytokines among three groups $(P<0.05)$. Compared with the control group, rats in the colitis group exhibited linear growth failure, as well as higher expression of calprotectin, tumour necrosis factor-a, interleukin- 6 and insulin-like growth factor binding protein 2, lower insulin-like growth factor-1 and insulin-like growth factor binding protein 3, and lower expression of nuclear factor kappa B in hepatocytes.

Conclusions: In addition to undernutrition, the systemic inflammatory response caused by asymptomatic colitis may inhibit the linear growth of rats by its influence on the insulin-like growth factor/insulin-like growth factor binding protein axis.

Keywords: Growth retardation, Asymptomatic colitis, Hormone, Cytokine, Rat

\section{Background}

It is widely recognized that infants and young children in impoverished areas of developing countries have persistently high rates of growth stunting, and undernutrition has long been considered the most important cause. However, recently, it has been increasingly realized that, although an adequate supply of energy and nutrients is clearly necessary, it is not sufficient to ensure normal

\footnotetext{
* Correspondence: majingqiu@xinhuamed.com.cn

${ }^{3}$ Shanghai Institute for Pediatric Research, Xinhua Hospital, School of Medicine, Shanghai Jiao Tong University, Shanghai Key Laboratory of Pediatric Gastroenterology and Nutrition, No.1665, Kongjiang Road, Yangpu District, Shanghai 200092, China

Full list of author information is available at the end of the article
}

linear growth [1]. Furthermore, some recent studies performed in poor areas have indicated the combined usage of zinc and multiple micronutrients did not improve growth faltering in infants and children $[2,3]$. Children aged less than 2 years, whose growth is rapid, are particularly vulnerable to environmental factors. Infants and young children living in conditions of poor sanitation are frequently exposed to pathogenic microbes, resulting in a substantial underlying burden of chronic and asymptomatic intestinal infection $[4,5]$.

The systemic inflammatory process induced by intestinal infection may suppress the growth hormone (GH)/insulinlike growth factor-1 (IGF-1)/IGF binding protein (IGFBP)

(C) The Author(s). 2019 Open Access This article is distributed under the terms of the Creative Commons Attribution 4.0 International License (http://creativecommons.org/licenses/by/4.0/), which permits unrestricted use, distribution, and 
axis, which is essential for normal linear growth, and may also have an impact on the effect of complementary feeding interventions in infants and young children [6]. However, the role of pro-inflammatory cytokines abnormally elevated during inflammation in disturbing the GH/IGF-1/IGFBP axis remains inconsistent [7-10]. Furthermore, a previous study of our research group carried out in Xichou County, Yunnan Province, China, a rural community, found that the length-for-age $\mathrm{Z}$ score (LAZ) of 6-month-old infants was substantially negative and that 1 year of nutritional intervention did not improve the growth faltering of rural infants [11]. Notably, the concentrations of faecal calprotectin, an established noninvasive biomarker of intestinal inflammation [12, 13], were increased significantly in rural healthy infants and were negatively correlated with the LAZ [14]. It was suggested that calprotectin may play a role in growth faltering. However, thus far, no study has explored the role of calprotectin in inhibiting growth and its mechanism.

A previous study concluded that inflammation itself has a harmful effect on linear growth, likely due, in part, to a reduction in the plasma concentrations of IGF-1 through a rat colitis model induced by trinitrobenzenesulphonic acid (TNBS) [15]. Nevertheless, the establishment of a colitis model was based on inflammatory bowel disease (IBD), a group of intestinal diseases with obvious clinical symptoms such as diarrhoea, pain and weight loss. Another murine intestinal infection model induced by Citrobacter rodentium suggested that linear growth failure was associated with systemic inflammation and suppressed serum levels of IGF-1. However, all mice with infectious colitis exhibited obvious diarrhoea during the experiment [16].

In our study, we established a rat model through TNBS enema in which rats had asymptomatic colitis similar to the potential intestinal infection caused by poor sanitation in children living in poverty areas. Using the rat model of asymptomatic colitis, the aims of our study were to explore 1) linear growth failure of rats, 2) changes in the faecal calprotectin and plasma levels of hormone and systemic cytokines, and 3) the relationship among linear growth, plasma hormone and cytokines.

\section{Methods}

\section{Animals}

Forty-eight 3-week-old male specific-pathogen-free Wistar rats (approximately $75 \mathrm{~g}$ to $95 \mathrm{~g}$ in body weight) were used in this study and were purchased from Shanghai SLAC Laboratory Animal Co., Ltd. (Shanghai, China). The rats were housed individually at an ambient temperature of $(22 \pm 1)^{\circ} \mathrm{C}$, were maintained under a 12-h light-dark cycle and were given standard laboratory chow and tap water. All the rats were anaesthetized by intraperitoneal injection of $1 \%$ pentobarbital sodium
$(0.01 \mathrm{~mL} / \mathrm{g})$ (Sigma Chemical Co., UK) before each operation to minimize animal suffering.

\section{Colitis model}

The rats were randomly divided into three groups $(n=$ 16/group): 1) free-feeding sham-operation control group (abbreviated as the control group), 2) 2,4,6-trinitrobenzenesulphonic acid (TNBS) colitis group (abbreviated as the colitis group) and 3) pair-fed sham-operation group (abbreviated as the pair-fed group). Rats in the control group were allowed free access to food, while the daily food intake of the pair-fed group matched that of their pairs in the colitis group. To achieve the precise matching of food intake, the pair-fed group was started 1 day after the colitis group [15]. Colitis was induced by the children's stomach tube inserted $5 \mathrm{~cm}$ proximal to the anus together with a little glycerol as lubricant. After the rats were anaesthetized by pentobarbital sodium $(1 \%$, $0.01 \mathrm{~mL} / \mathrm{g}$ ), a volume of $0.1 \mathrm{~mL}$ of a solution of $2.5 \%$ TNBS (5\% TNBS; Sigma Chemical Co. UK; half diluted in Phosphate Buffered Saline (PBS)) was injected into the colon, and this step was repeated every 2 days in the colitis group. In the control and pair-fed groups, a volume of $0.1 \mathrm{~mL}$ of PBS (0.01 M, pH 7.4) was injected into the colon as the sham operation. The rats were measured for their weight and length on the operation day (marked as d0), 3rd day (marked as d3) and 5th day (marked as $\mathrm{d} 5$ ). The rats' body length, assessed by the mean of two measurements of the nose-to-tail base distance, was measured after being anaesthetized before sacrifice [15]. The previous $24 \mathrm{~h}$ of food intake was measured daily in all rats.

At $\mathrm{d} 3$ and $\mathrm{d} 5,24$ rats (8 for each group) were anaesthetized by a dose of pentobarbital sodium (1\%, 0.015 $\mathrm{mL} / \mathrm{g}$ ). The rats were fixed in the supine position, and approximately $1.5 \mathrm{~mL}$ of blood from the heart was collected into EDTA tubes. Next, each rat was perfused transcardially with $150 \mathrm{~mL}$ of PBS, followed by $200 \mathrm{~mL}$ of $4 \%$ paraformaldehyde in $0.01 \mathrm{M}$ phosphate buffer $(\mathrm{pH}$ 7.4). During this perfusion process, the rats were mercifully killed without any pain. The blood was centrifuged at $4{ }^{\circ} \mathrm{C}$ at a speed of $3000 \mathrm{rpm}$ for $15 \mathrm{~min}$, and the plasma was stored at $-80^{\circ} \mathrm{C}$ until ELISA. Using midline laparotomy, approximately $0.5 \mathrm{~cm}^{3}$ of the liver tissue was fixed in $4 \%$ ice-cold paraformaldehyde for $24 \mathrm{~h}$ for immunohistochemistry. The $7-\mathrm{cm}$ colon proximal to the anus was removed and opened longitudinally, and faeces in the colon section were stored at $-20^{\circ} \mathrm{C}$ for calprotectin detection. After the colon section was rinsed with PBS and exposed, macroscopic inflammation was assessed, and then the colon was cut longitudinally into two halves, one half was weighed and five times the volume of RIPA (Thermo) was added for homogenization. The colon homogenate was first centrifuged for $20 \mathrm{~min}$ 
at $2100 \mathrm{rpm}$, and then the supernatant was recentrifuged for $15 \mathrm{~min}$ at $12000 \mathrm{rpm}$ and stored at $-80^{\circ} \mathrm{C}$ until assayed for myeloperoxidase (MPO) concentrations. The remaining half of the colon was fixed in $4 \%$ ice-cold paraformaldehyde for $24 \mathrm{~h}$ for histopathologic staining. Our animal experiments complied with the ARRIVE guidelines.

\section{Macroscopic score}

Macroscopic damage of the colonic mucosa was assessed by two independent observers who were unaware of the animals' treatment. The scale for macroscopic damage ranged from 0 (normal) to 5 (severe) and was based on hyperaemia, sites of ulceration, and sites of inflammation according to the criteria of Morris (Additional file 1: Table S1) [17].

\section{Pathology and immunohistochemistry}

The rats' colon samples were fixed in $4 \%$ paraformaldehyde, embedded in paraffin, sectioned and stained with haematoxylin and eosin (HE).

Immunohistochemical staining of paraffin-embedded liver tissues was performed by incubating with the primary antibodies anti-rat growth hormone receptor (GHR) (\#119-12,770; 1:100 dilution; RayBiotech) and nuclear factor kappa B (NF-kB) p65 (\#ab13594; 1:100 dilution; Abcam) overnight at $4{ }^{\circ} \mathrm{C}$. After the incubation with the biotin-conjugated 2nd antibody for 2 hours at room temperature, the samples were treated with $A B C$ Elite immunoperoxidase (Vector Laboratories) and $\mathrm{DAB}$ substrate (Vector Laboratories) according to the manufacturers' instructions and were finally stained with haematoxylin. The analysis and semi-quantification of positive immunostaining were performed using ImagePro Plus v7.0 software (Media Cybernetics Inc., USA) using the parameter of the mean density. All images were captured using a CoolSNAP colour camera (Media Cybernetics Inc., USA) at a magnification of $200 \times$ in an Olympus BX51 microscope.

\section{Myeloperoxidase (MPO) concentrations}

The tissue concentrations of MPO were measured to assess the degree of intestinal inflammation using the MPO colorimetric activity assay kit (\#MAK068; SigmaAldrich, St. Louis, USA). Fifty microliters of 1:40-diluted colon homogeneity samples was added to 50 microliters of MPO substrate according to the manufacturer's instructions. The reaction was stopped after $60 \mathrm{~min}$ at room temperature away from light, and the absorbance at $412 \mathrm{~nm}$ was measured by the microplate reader (Molecular Device, USA). The amount of TNB consumed by the enzyme assay were determined by the difference value between each sample blank and its corresponding samples $\left(\Delta \mathrm{A}_{412}=(\mathrm{A} 412)_{\text {sample blank }}-(\mathrm{A} 412)_{\text {sample }}\right.$, then we should compare the $\Delta \mathrm{A}_{412}$ of each sample to the standard curve to get the amount of TNB consumed. The $\mathrm{MPO}$ activity was calculated by the following equation:

$$
\text { MPO activity }=\frac{B \times \text { Sample Dilution Factor }}{(\text { Reaction Time }) \times \mathrm{V}}
$$

where $\mathrm{B}=$ amount of chromophore TNB consumed, and $\mathrm{V}=$ sample volume added to well. All the samples were analysed in 3 wells in each experiment, and each experiment was repeated 3 times.

\section{Elisa}

The concentrations of plasma and faecal calprotectin (\#K6936; Immundiagnostik, Bensheim, Germany), plasma concentrations of IL-6 (\#EZRIL6; Millipore, Boston, MA, USA), TNF- $\alpha$ (\#ELR-TNFa; RayBiotech, Norcross, GA, USA), IGF-1 (\#ELR-IGF1; RayBiotech, Norcross, GA, USA), IGF-binding protein-3 (IGFBP3) (\#80581; Crystal Chem, Downers Grove, USA), IGFBP2 (\#ab207615; Abcam, Cambridge, MA, USA) and GH (\#EZRMGH-45 K; Millipore, Boston, MA, USA) were determined by ELISA. All the experiments were performed according to the manufacturer's instructions.

\section{Statistical analysis}

SPSS 13.0 statistical software was used for all descriptive analyses. The results were presented as the means \pm SD for normally distributed continuous variables or medians (interquartile range) for data not normally distributed. Linear growth was presented as the change in the body length during the five-day experimental period. Independent two-sample t-test and one-way ANOVA were used to compare normally distributed continuous variables among the experimental groups, and skewed data were compared after log transformations by ANOVA. The LSD multiple comparison test was used for the post hoc test if the overall ANOVA had a $P$ value less than 0.05 . Chi-square test was used for categorical variables. Pearson's correlation was used to test for a linear relationship between two quantitative variables (skewed data were analysed after $\log$ transformation). All statistical tests were two-tailed, and $P$ values $<0.05$ were considered statistically significant.

\section{Results}

\section{General conditions}

The rats in the three groups appeared well, with a flexible response, glossy hair, active foraging and no significant reduction in activity. They all had solid stools and no diarrhoea throughout the course of experiment. There was no death in all rats. 


\section{Macroscopic and pathological findings}

The macroscopic findings showed hyperaemia and oedema in the colonic mucosa in the rats of both the colitis and pair-fed groups, suggesting a low level of colonic damage (score $\leq 1)$. There was no detectable macroscopic injury in the colons of rats from the control group.

The pathological findings showed that the colonic villus was intact after HE staining in all three groups. In both the colitis and pair-fed groups, the basal layer under the epithelium was obviously loose and the space between the muscle and epithelium layer was larger than that in the control group (Fig. 1a, b). Hemangiectasis was found in both the colitis and pair-fed groups at $\mathrm{d} 3$ and d5 (Fig. 1a).

\section{Myeloperoxidase (MPO) in colonic tissue}

The MPO activity is an indicator of the infiltration of the colon with polymorphonuclear leukocytes caused by the TNBS. There were significant differences in the colonic MPO concentrations among the three groups at $\mathrm{d} 3$ and $\mathrm{d} 5(F=34.49, P<0.001 ; F=23.67, P<0.001)$, and the colonic MPO concentrations in the colitis group were higher than those in the control group or pair-fed group $(P<0.01)$ (Fig. 2). However, the difference in the MPO concentrations between the control and pair-fed groups was not significant $(P>0.05)$ (Fig. 2$)$.

\section{Comparison of the cytokine levels among the three groups}

At $\mathrm{d} 3$ and $\mathrm{d} 5$, the faecal calprotectin concentrations among the three groups were significantly different $(F=$ $19.09, P<0.001 ; F=4.35, P=0.026)$. At $\mathrm{d} 3$, the faecal calprotectin levels were increased in both the colitis and pair-fed groups compared with those in the control group $(P<0.05)$, and the rats in the pair-fed group had significantly higher faecal calprotectin levels than those in the colitis group $(P<0.05)$. At $\mathrm{d} 5$, the faecal calprotectin levels in the pair-fed group were significantly higher than those in the control group $(P<0.05)$ (Fig. 2).

At d3 and d5, the plasma calprotectin concentrations among three groups were significantly different $(F=$ 5.92, $P=0.009 ; F=7.16, P=0.004$ ) and were significantly higher in the colitis and pair-fed groups than in the control group $(P<0.05)$ (Fig. 2).

At $d 3$ and $d 5$, there were significant differences in the plasma TNF- $\alpha$ concentrations among the three groups $(F=4.44, P=0.025 ; F=20.02, P<0.001)$ that were significantly higher in the colitis and pair-fed groups than in the control group $(P<0.05)$ at $\mathrm{d} 3$ (Fig. 2$)$. At $\mathrm{d} 5$, the TNF- $\alpha$ concentrations were increased in the pair-fed group compared with those at $\mathrm{d} 3(t=2.79, P=0.014)$ and were significantly higher than those in the colitis and control groups $(P<0.05)$. Furthermore, the rats in the colitis group had significantly higher TNF- $\alpha$ concentrations than those in the control group $(P<0.05)$ at $\mathrm{d} 5$ (Fig. 2).

At $\mathrm{d} 3$ and $\mathrm{d} 5$, significant differences were found in the plasma IL-6 concentrations among the three groups $(F=6.07, P=0.008 ; F=3.90, P=0.036)$, and the rats in the colitis and pair-fed groups had significantly higher IL- 6 concentrations than those in the control group $(P<$ 0.05) (Fig. 2).

\section{Food intake}

The daily food intake of rats in the pair-fed group was matched to the pair in the colitis group; thus, the food

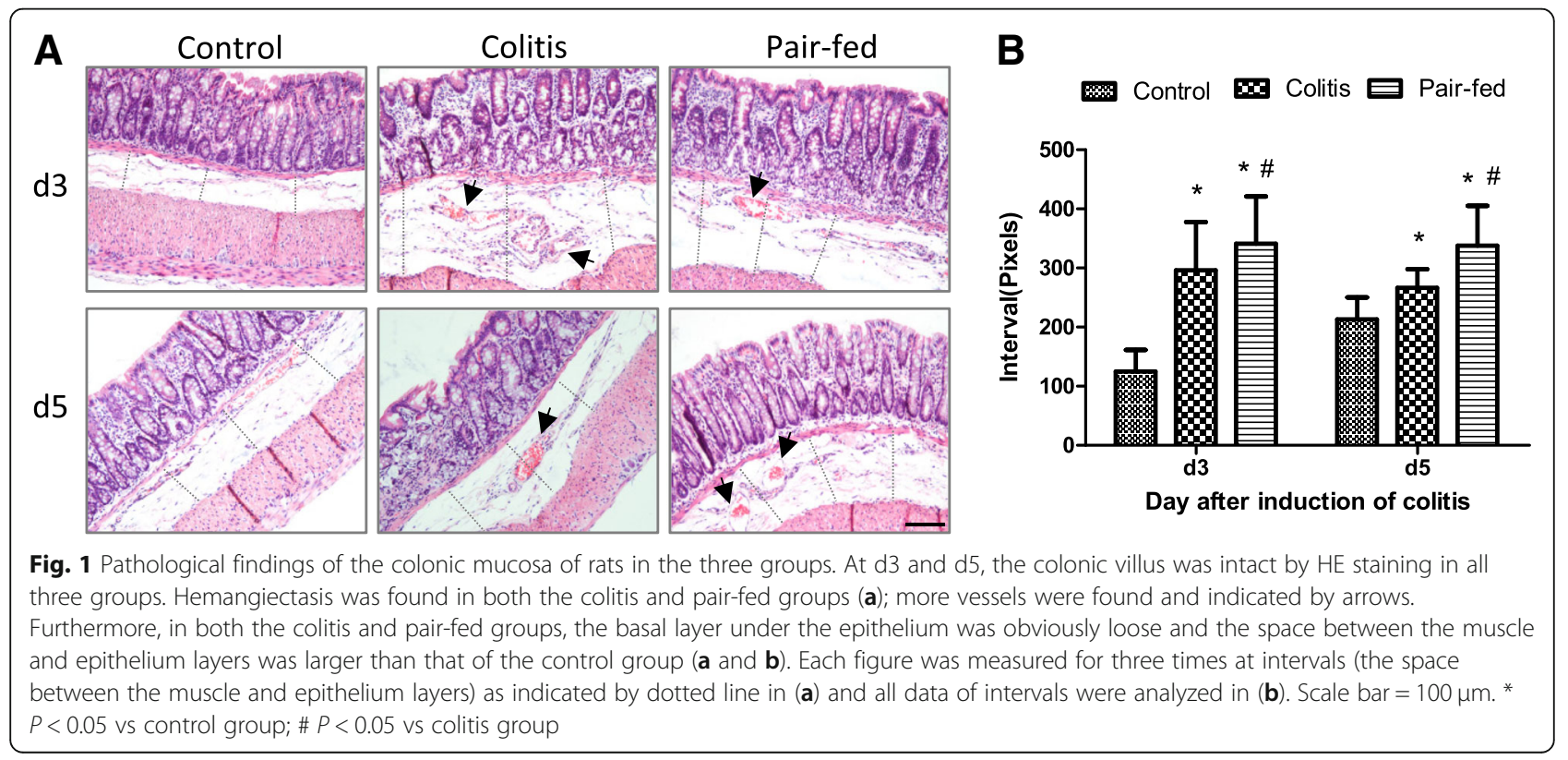




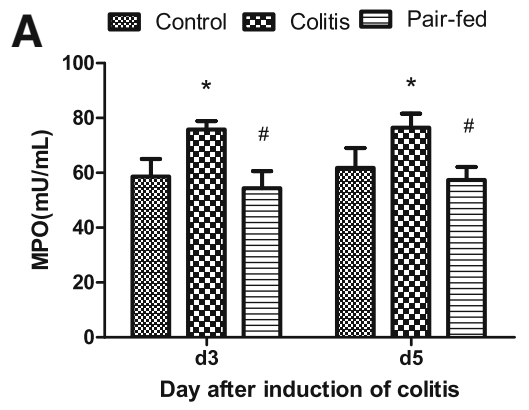

B Control E Colitis

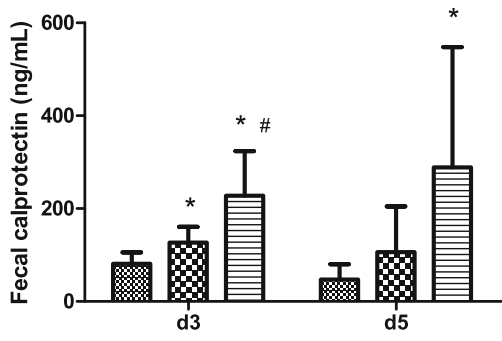

Day after induction of colitis

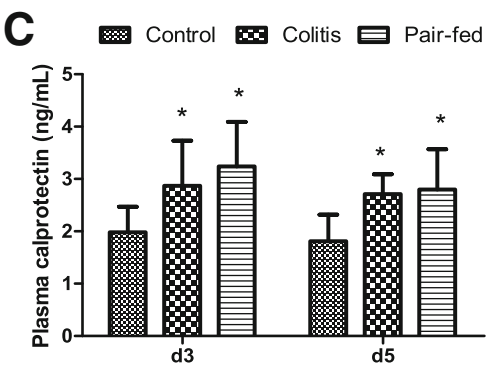

Day after induction of colitis
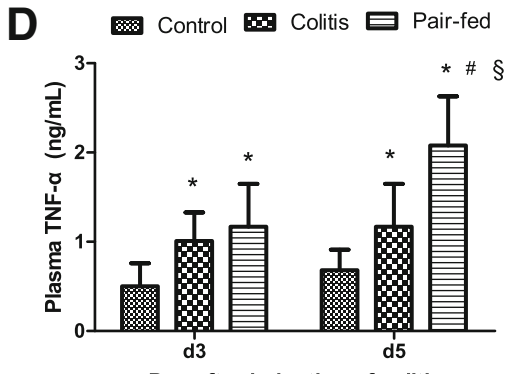

Day after induction of colitis

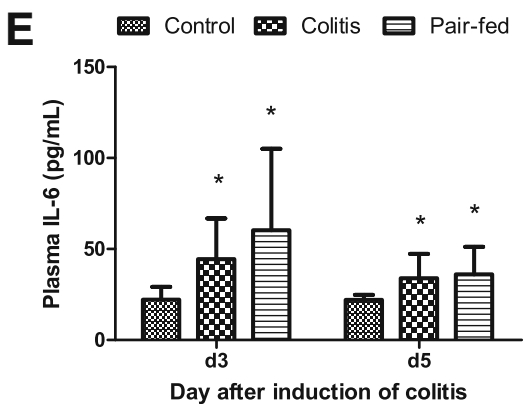

Fig. 2 Comparison of MPO in colonic tissue and cytokine levels among the three groups at $\mathrm{d} 3$ and $\mathrm{d} 5$. The values are expressed as the means (SD), $n=8$ /group. a Intestinal MPO concentration. $\mathbf{b}$ Faecal calprotectin levels. c Plasma calprotectin concentrations. d Plasma TNF-a concentrations. e Plasma IL-6 concentrations. The skewed data of faecal calprotectin, TNF- $a$ and IL-6 were compared after log transformations among the three groups by ANOVA. ${ }^{*} P<$ 0.05 vs control group; \# $P<0.05$ vs colitis group; $\S P<0.05$ vs d3

intake was only compared between the control and colitis groups.

The food intake of rats in the control group was increased gradually over time. However, the food intake in the colitis group was increased slowly after enema and was decreased obviously at $\mathrm{d} 3$. The rats in the colitis group ate significantly less than those in the control group at $\mathrm{d} 2, \mathrm{~d} 3$ and $\mathrm{d} 5(t=2.51, P=0.018 ; t=4.74, P<$ $0.001 ; t=3.22, P=0.006$ ) (Fig. 3 ).

\section{Weight gain and linear growth of rats}

At $\mathrm{d} 3$ and $\mathrm{d} 5$, the weight gain (weight at $\mathrm{d} 3$ or $\mathrm{d} 5$ subtracts weight at $\mathrm{d} 0$ ) was significantly different among the three groups $(F=3.94, P=0.026 ; F=19.22, P<0.001)$ and the weight gain in the colitis group and pair-fed group was significantly less than that in the control group $(P<0.05)$ (Fig. 3$)$.

At $\mathrm{d} 5$, the increases in the body length of the control, colitis and pair-fed groups were $1.65 \pm 0.34 \mathrm{~cm}, 1.10 \pm$ $0.30 \mathrm{~cm}$ and $1.38 \pm 0.26 \mathrm{~cm}$, respectively, and there was a significant difference among the three groups $(F=6.55$, $P=0.006)$. The increase in the body length of rats in the colitis group was significantly less than that in the control group $(P<0.05)$ (Fig. 3$)$.

\section{Plasma hormone concentrations}

There was no significant difference in the plasma $\mathrm{GH}$ concentrations among the three groups at $\mathrm{d} 3$ and $\mathrm{d} 5$ $(P=0.173 ; P=0.082)$ (Fig. 4).

At $\mathrm{d} 3$ and $\mathrm{d} 5$, there were significant differences in the plasma IGF-1 concentrations among the three groups $(F=6.12, P=0.008 ; F=4.29, P=0.027)$. At d3, the IGF1 concentrations were significantly lower in the colitis group than those in the control group and pair-fed group $(P<0.05)$ (Fig. 4). At d5, the IGF-1 concentrations in both the colitis and pair-fed groups were further decreased compared with those at $\mathrm{d} 3(t=2.89, P=0.012$; $t=3.42, P=0.004)$ and were significantly lower than those in the control group $(P<0.05)($ Fig. 4).

The plasma IGFBP3 concentrations were significantly different among the three groups at $\mathrm{d} 3$ and $\mathrm{d} 5(F=$ 13.22, $P<0.001 ; F=24.76, P<0.001)$ and were significantly lower in both the colitis and pair-fed groups than those in the control group $(P<0.05)$ (Fig. 4). At d3 and $\mathrm{d} 5$, the plasma IGFBP2 concentrations were significantly 


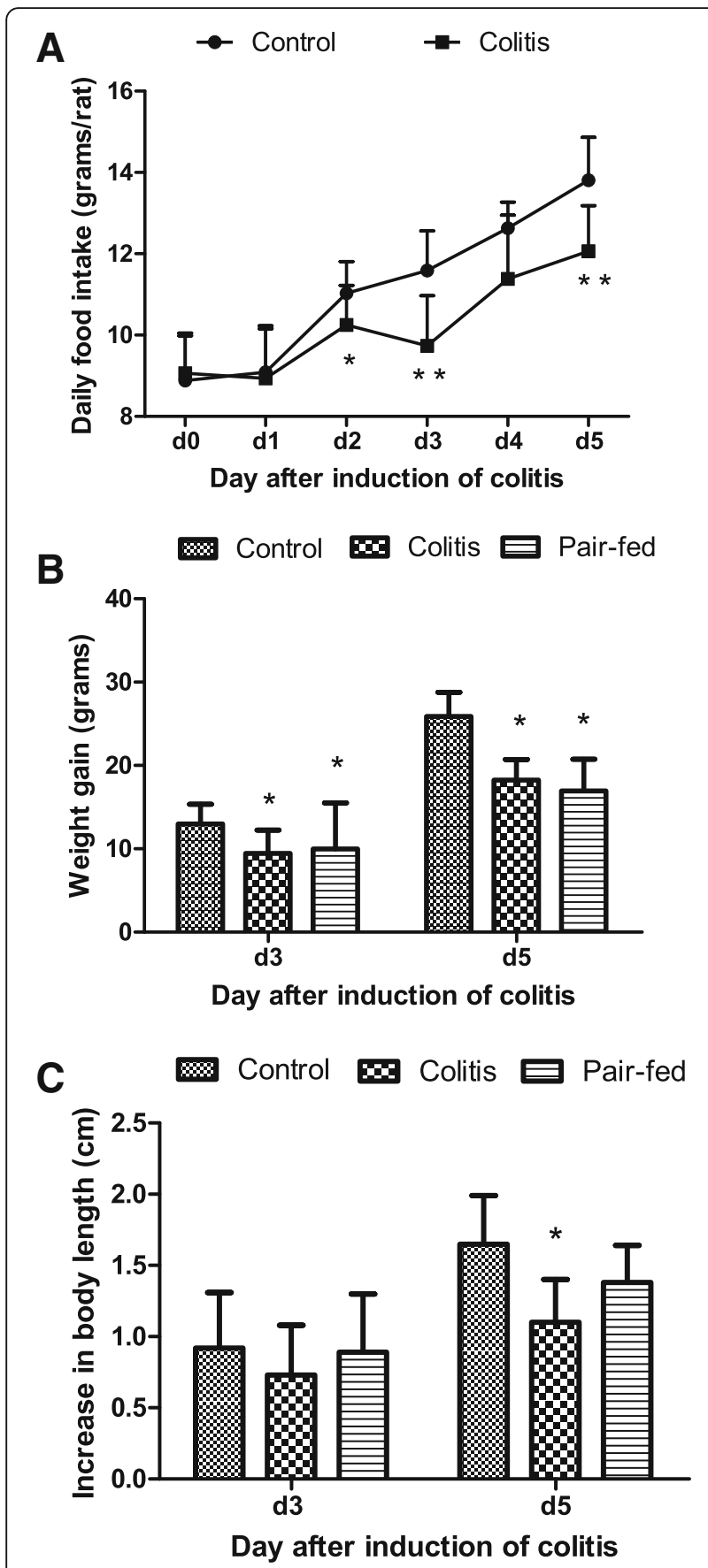

Fig. 3 Comparison of the daily food intake, weight gain and increase in the body length among the three groups. The values are expressed as the means (SD). a Daily food intake. b Weight gain. $\mathbf{c}$ Increase in body length. ${ }^{*} P<0.05$ vs control group; ${ }^{*} P<0.01$ vs control group. By design, food intake in the pair-fed group was the same as that in the colitis group

different among the three groups $(F=19.90, P<0.001$; $F=15.80, P<0.001)$ and were significantly higher in both the colitis and pair-fed groups than those in the control group $(P<0.05)$. Additionally, the rats in the pair-fed group had significantly higher IGFBP2 concentrations than those in the colitis group $(P<0.05)$ (Fig. 4$)$.

\section{Relationship among linear growth, plasma hormone and cytokines}

The relationship among linear growth, plasma hormone and cytokines of all 48 rats were analysed. The results of Pearson's correlation indicated that both the faecal and plasma concentrations of calprotectin were negatively correlated with the increase in the body length at $\mathrm{d} 5$ (respectively: $r=-0.501, P=0.013 ; r=-0.469, P=0.021$ ). Furthermore, at $\mathrm{d} 5$, the TNF- $\alpha$ concentrations were negatively correlated with the IGF-1 concentrations $(r=$ $-0.424, P=0.039$ ). At $\mathrm{d} 5$, the IGFBP2 concentrations were positively correlated with the plasma concentrations of IL-6, TNF- $\alpha$ and calprotectin (respectively: $r=$ 0.461, $P=0.023 ; r=0.629, P=0.001 ; r=0.488, P=0.016$ ), while the IGFBP3 concentrations were negatively correlated with the plasma concentrations of IL-6, TNF- $\alpha$ and calprotectin and faecal concentrations of calprotectin (respectively: $r=-0.630, P=0.001 ; r=-0.801, P<0.001$; $r=-0.711, P<0.001 ; r=-0.593, P=0.002)$.

The IGF-1 concentrations were positively correlated with the increase in the body length at $\mathrm{d} 5(r=0.419, P=$ 0.042). At $\mathrm{d} 3$, it was also found that the calprotectin concentrations in faeces were positively correlated with those in plasma $(r=0.532, P=0.007)$.

Expression of NF-KB and GHR proteins in hepatocytes, as determined by immunohistochemistry

The expression of NF- $\mathrm{kB}$ proteins (mean density) in hepatocytes was significantly different among the three groups at $\mathrm{d} 3(F=9.31, P=0.006)$ and was significantly lower in both the colitis and pair-fed groups than in the control group $(P<0.05)$. Furthermore, NF- $\mathrm{kB}$ protein expression was similar between the colitis and pair-fed groups $(P>0.05)$. At $\mathrm{d} 5$, NF- $\mathrm{kB}$ protein expression was increased in both the colitis and pair-fed groups, and no significant difference was found in the NF- $\mathrm{kB}$ protein expression among the three groups $(P>0.05)$. No significant differences were found in the expression of GHR proteins in hepatocytes among the three groups $(P>$ 0.05 ) at $\mathrm{d} 3$ and $\mathrm{d} 5$ (Fig. 5).

At $\mathrm{d} 3$, NF- $\mathrm{kB}$ protein expression was negatively correlated with the plasma concentrations of TNF- $\alpha$ and faecal concentrations of calprotectin (respectively: $r=-$ $0.678, P=0.015 ; r=-0.631, P=0.028)$.

\section{Discussion}

In order to mimic asymptomatic intestinal inflammation in children in poor areas caused by poor sanitation, based on the results of preliminary experiments, in this study, we established the asymptomatic colitis model using a $2.5 \%$ low enema concentration of TNBS, approximately 

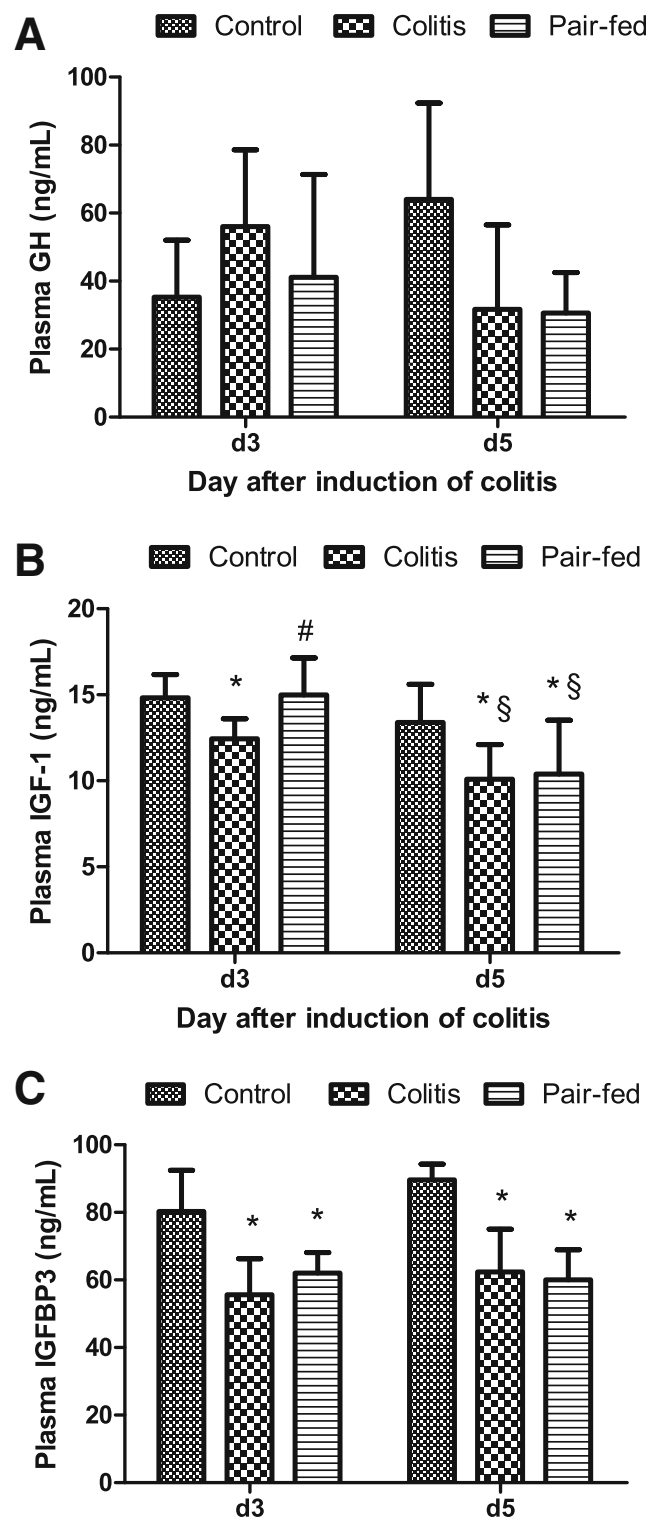

Day after induction of colitis
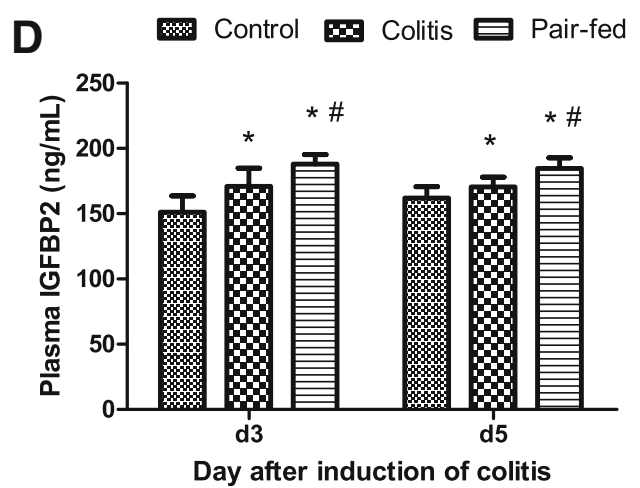

Fig. 4 Comparison of the plasma hormone concentrations among the three groups at $d 3$ and $d 5$. The values are expressed as the means (SD), $n=8$ /group. a Plasma GH. b Plasma IGF-1. c Plasma IGFBP3. d Plasma IGFBP2. Skewed data of plasma GH are compared after log transformations among three groups by ANOVA. ${ }^{*} P<0.05$ vs control group; \# $P<0.05$ vs colitis group; $§ P<0.05$ vs d3

half of the TNBS concentrations used in other colitis models $[15,18]$. Furthermore, in order to reduce the severity of intestinal inflammation, we did not use ethanol as the "barrier breaker", but increased the frequency of TNBS enema (every 2 days). Thus, no ulcer and only hyperaemia and oedema were found in the colonic mucosa of the colitis group by macroscopic observation. Pathological findings also indicated only mild damage in the colonic mucosa. The colonic MPO concentrations in the colitis group were significantly increased. Moreover, we found an obvious decrease in the body length increase and less weight gain 5 days after TNBS enema compared with that in the control group. Therefore, this model may serve as a novel means of assessing the mechanisms of linear growth suppression during asymptomatic colitis.

In the model, the sensitive biomarker of intestinal inflammation, faecal calprotectin, was significantly increased after TNBS administration, indicating a certain degree of colonic inflammation occurred in the colitis model. Furthermore, the plasma levels of calprotectin, TNF- $\alpha$ and IL-6 were also significantly increased, suggesting a systemic inflammatory response in the rats. An increased plasma concentration of calprotectin (also called S100A8/ A9) correlates well with disease activity in many inflammatory conditions and is already accepted as a superior biomarker to other inflammation markers such as Creactive protein (CRP) $[19,20]$. Furthermore, calprotectin is not only a useful marker of inflammation but also plays a pivotal role in the pathogenesis of inflammatory disorders $[21,22]$. The concentration of calprotectin in faeces is approximately 6 times that in plasma in healthy adults [23]. In this study, a positive correlation was also found between the calprotectin concentrations in faeces and plasma. Faecal calprotectin has been widely used in the detection of intestinal inflammation. Due to the simplicity of collection, storage and detection, compared with plasma calprotectin, faecal calprotectin has the special advantage in infants and young children of not requiring venepuncture. In a previous study, we found a significantly negative correlation between faecal calprotectin and linear growth in rural infants [14]. In this study, we confirmed this relationship again by establishing animal models. It was suggested that the elevated calprotectin may have a direct inhibitory effect on the linear growth of children. Therefore, it is necessary to further explore the mechanism of calprotectin inhibiting growth, in the hope that faecal calprotectin will be used as an important screening 
indicator in the future to identify intestinal inflammationinduced growth retardation for sub-healthy children in poor rural areas.

Normal growth is largely dependent on the GH-IGFIGFBP axis, and any factor impairing the GH-IGF-IGFBP axis will produce adverse effects on linear growth. To explore the relationship between systemic inflammation and linear growth, the plasma levels of GH, IGF-1, IGFBP2 and IGFBP3 were detected, and further expression of NF$\kappa \mathrm{B}$ and GHR proteins in hepatocytes were also determined. Thus, we found that the IGF-1 level in rats in the colitis group decreased significantly at $\mathrm{d} 3$ and further decreased at d5. Moreover, we observed a transient decrease in NF- $\mathrm{kB}$ protein expression in hepatocytes in the colitis group that was negatively correlated with the faecal calprotectin concentrations at $\mathrm{d} 3$. NF- $\mathrm{kB}$ is a critical signalling molecule in the inflammatory process that not only exerts a pro-inflammatory effect but also plays an antiinflammatory role [24] and may mediate the inhibitory effect of TNF- $\alpha$ on IGF $[25,26]$. Our results suggested that calprotectin might have an inhibitory effect on IGF-1 production via the NF- $\mathrm{kB}$-mediated signalling pathway.

Additionally, both decreased IGFBP3 and increased IGFBP2 levels were found in the colitis group, and the levels of two binding proteins were significantly correlated with calprotectin. Most of the circulating IGF-1 protein is in complex with acid labile subunit (ALS) and IGFBP-3, which increases its half-life by 100 fold. Elevated inflammatory cytokines may increase the proteolysis of IGFBP-3 and impair the formation of the IGF-1/IGFBP-3/ALS complex, resulting in a shorter IGF-1 half-life and enhanced clearance of IGF-1 [7]. Furthermore, increased levels of IGFBP2 have previously been described in paediatric IBD patients [27]. The mitogenic effect of IGF-1 was strongly reduced by the exogenous administration of IGFBP-2 in human growth plate chondrocytes [28]. Thus, the reduction in the IGF-1 concentration may be partly attributed to the influence of abnormally elevated calprotectin on IGFBP3 and IGFBP2.

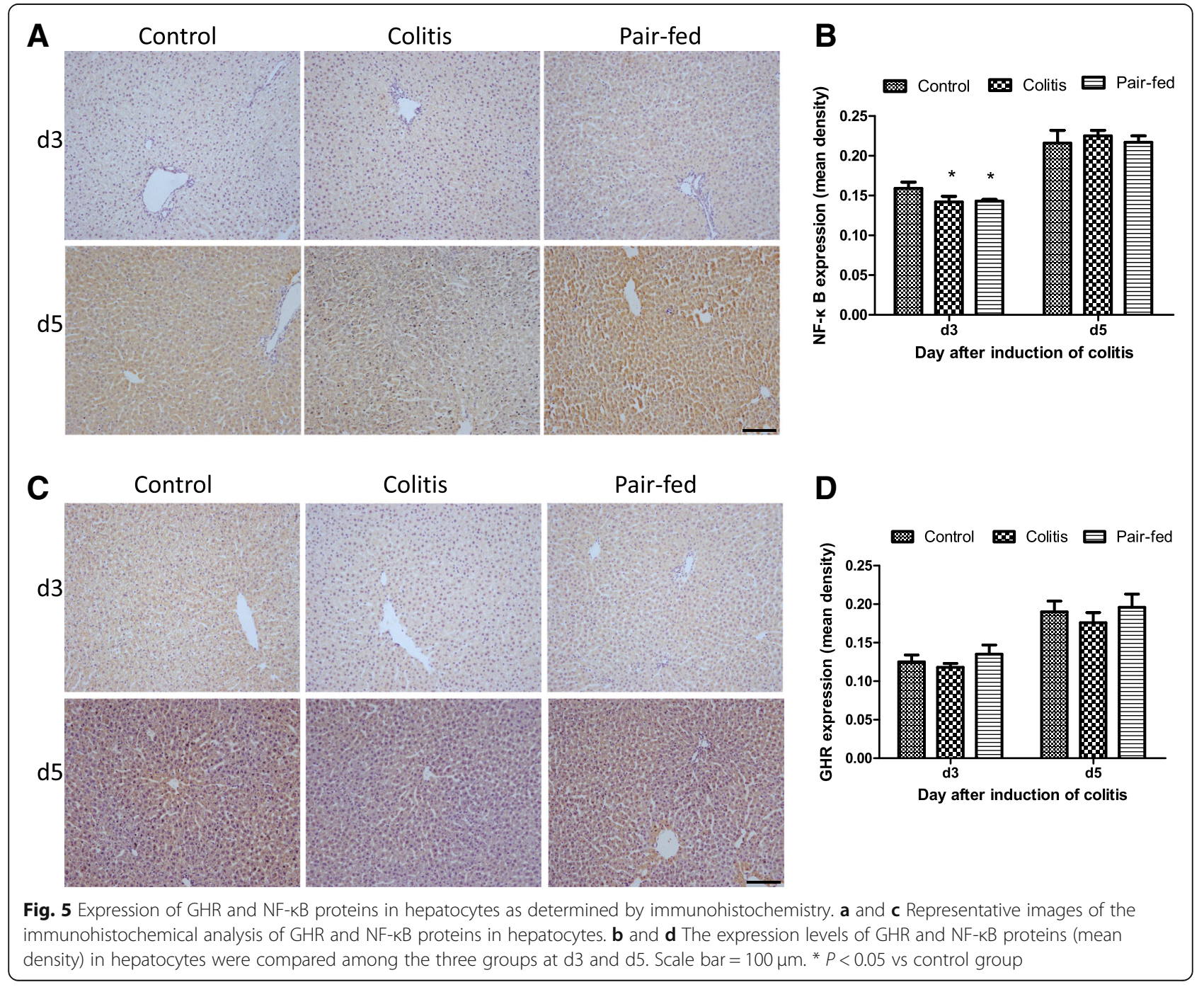




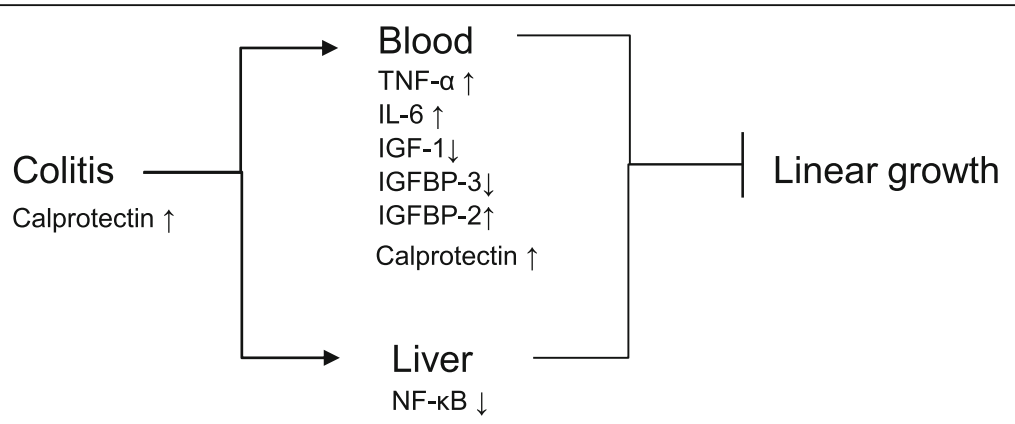

Fig. 6 Systemic inflammatory response induced by asymptomatic colitis may partly cause linear growth retardation by the influence on the IGF/IGFBP axis

However, a significant correlation between two variables does not indicate a cause-and-effect relationship. In future research we will use antibody blockade method to further elucidate underlying mechanisms of calprotectin inhibiting linear growth.

In our study, elevated IL- 6 and TNF- $\alpha$ may also inhibit the linear growth of rats by the influence on IGFBP2 and IGFBP3. Moreover, the negative correlation between TNF- $\alpha$ and IGF- 1 has indicated TNF- $\alpha$ might have a direct inhibitory effect on IGF-1, similar to that observed in other animal studies $[29,30]$.

Animal studies have shown that inflammatory cytokines could induce hepatic GH resistance $[7,8]$. However, as opposed to $\mathrm{GH}$ resistance, $\mathrm{GH}$ secretion was also reported to be normal or low in children with inflammatory growth retardation in IBD [31]. In our study, there was no GH resistance associated with elevated inflammatory cytokines.

Additionally, the colonic mucosa of the rats in the pairfed group also showed a similar and pathological manifestation to that of the colitis group that may be attributed to the inadequate intake of proteins and carbohydrates. However, TNF- $\alpha$ and faecal calprotectin in the pair-fed group were increased as high as or even higher than those in the colitis group. Thus, we further evaluated the expression of autophagy marker protein light chain 3 (LC3) in hepatocytes [32] by immunohistochemistry and found it was increased in the pair-fed group compared with that in other two groups, although no significant difference was found among the three groups $(P=0.081$, data not shown) . In view of this, the involvement of starvation-induced autophagy should be considered [33].

\section{Conclusions}

This study was the first to establish an asymptomatic colitis model mimicking the growth pattern observed in early childhood in poor areas where the children are at high risk of intestinal inflammation. Furthermore, this study is the first to explore the relationship among linear growth, plasma hormone and calprotectin. In addition to the undernutrition, the systemic inflammatory response caused by asymptomatic colitis may also aggravate the linear growth retardation by the influence on the IGF/ IGFBP axis (Fig. 6).

\section{Additional file}

Additional file 1: Table S1. Criteria for assessment of colonic damage induced by TNBS. (DOCX $16 \mathrm{~kb})$

\section{Abbreviations \\ CRP: C-reactive protein; GH: Growth hormone; GHR: Growth hormone receptor; IBD: Inflammatory bowel disease; IGF-1: Insulin-like growth factor-1; IGFBP2: Insulin-like growth factor binding protein 2; IGFBP3: Insulin-like growth factor binding protein 3; IL-6: Interleukin-6; LAZ: Length-for-age Z score; MPO: Myeloperoxidase; NF-kB: Nuclear factor kappa B; \\ TNBS: Trinitrobenzenesulphonic acid; TNF-a: Tumour necrosis factor-a}

\section{Acknowledgements}

Not applicable.

Authors' contributions

XYS participated in the design, analysis of the research and drafting of the paper. XQS participated in the acquisition of data, analysis of data and drafting of the paper. FL and JLW participated in the acquisition of data. JQM participated in the design and analysis of the research and revision of the paper and had primary responsibility for the final content. All the authors have read and approved the final manuscript.

\section{Funding}

This study was funded by the National Natural Science Foundation of China (No. 81302446, No. 81172686 and No. 81703249). The funding sources were not involved in the preparation of the manuscript or in the collection, analysis or interpretation of the data.

\section{Availability of data and materials}

All data generated or analysed during this study are included in this published article.

\section{Ethics approval and consent to participate}

All the experimental procedures were carried out according to the Guide for the Care and Use of Laboratory Animals (eighth edition, published in 2011) by the National Research Council (USA), Committee for the Update of the Guide for the Care and Use of Laboratory Animals. This animal experiment was approved by the Ethics Committee of Xinhua Hospital Affiliated to Shanghai Jiao Tong University, School of Medicine (Approval No. XHEC-F2016-018).

Consent for publication Not applicable. 


\section{Competing interests}

The authors declare that they have no competing interests.

\section{Author details}

'Department of Children and Adolescents Health Care, Xin Hua Hospital, School of Medicine, Shanghai Jiao Tong University, Shanghai Institute for Pediatric Research, MOE-Shanghai Key Laboratory of Children's Environmental Health, No.1665, Kongjiang Road, Yangpu District, Shanghai 200092, China. ${ }^{2}$ Department of Biochemistry and Molecular Cell Biology, Shanghai Jiao Tong University School of Medicine, 280 S. South Chongqing Road, Shanghai 200025, China. ${ }^{3}$ Shanghai Institute for Pediatric Research, Xinhua Hospital, School of Medicine, Shanghai Jiao Tong University, Shanghai Key Laboratory of Pediatric Gastroenterology and Nutrition No.1665, Kongjiang Road, Yangpu District, Shanghai 200092, China.

Received: 5 December 2018 Accepted: 13 June 2019

Published online: 20 June 2019

\section{References}

1. Panter-Brick C, Lunn PG, Langford RM, Maharjan M, Manandhar DS. Pathways leading to early growth faltering: an investigation into the importance of mucosal damage and immunostimulation in different socioeconomic groups in Nepal. Br J Nutr. 2009;101:558-67 PMID: 18662426.

2. Wang AZ, Shulman RJ, Crocker AH, Thakwalakwa C, Maleta KM, Devaraj S, et al. A combined intervention of zinc, multiple micronutrients, and albendazole does not ameliorate environmental enteric dysfunction or stunting in rural Malawian children in a double-blind randomized controlled trial. J Nutr. 2017;147(1):97-103 PMID:27807040.

3. Locks LM, Manji KP, McDonald CM, Kupka R, Kisenge R, Aboud S, et al. Effect of zinc and multivitamin supplementation on the growth of Tanzanian children aged 6-84 wk: a randomized, placebo-controlled, double-blind trial. Am J Clin Nutr. 2016;103(3):910-8 PMID: 26817503

4. Kosek MN, MAL-ED Network Investigators. Causal pathways from enteropathogens to environmental enteropathy: findings from the MAL-ED birth cohort study. EBioMedicine. 2017:18:109-17 PMID:28396264.

5. Owino V, Ahmed T, Freemark M, Kelly P, Loy A, Manary M, et al. Environmental Enteric Dysfunction and Growth Failure/Stunting in Global Child Health. Pediatrics. 2016;138(6). https://doi.org/10.1542/peds.2016-0641 PMID:27940670.

6. Mbuya MN, Humphrey JH. Preventing environmental enteric dysfunction through improved water, sanitation and hygiene: an opportunity for stunting reduction in developing countries. Matern Child Nutr. 2016; 12(Suppl 1):106-20 PMID: 26542185.

7. Vortia E, Kay M, Wyllie R. The role of growth hormone and insulin-like growth factor-1 in Crohn's disease: implications for therapeutic use of human growth hormone in pediatric patients. Curr Opin Pediatr. 2011;23(5): 545-51 PMID 21900782.

8. Zhao $Y$, Xiao X, Frank SJ, Lin HY, Xia Y. Distinct mechanisms of induction of hepatic growth hormone resistance by endogenous IL-6, TNF- $a$, and IL-1 $\beta$. Am J Physiol Endocrinol Metab. 2014;307(2):E186-98 PMID: 24895283.

9. Ezri J, Marques-Vidal P, Nydegger A. Impact of disease and treatments on growth and puberty of pediatric patients with inflammatory bowel disease. Digestion. 2012;85(4):308-19 PMID: 22688404.

10. Gasparetto M, Guariso G. Crohn's disease and growth deficiency in children and adolescents. World J Gastroenterol. 2014;20(37):13219-33 PMID: 25309059.

11. Tang M, Sheng XY, Krebs NF, Hambidge KM. Meat as complementary food for older breastfed infants and toddlers: a randomized, controlled trial in rural China. Food Nutr Bull. 2014;35(4 Suppl):S188-92 PMID:25639137.

12. Zhu Q, Li F, Wang J, Shen L, Sheng X. Fecal calprotectin in healthy children aged 1-4 years. PLoS One. 2016;11(3):e0150725 PMID: 26950440

13. Li F, Ma J, Geng S, Wang J, Liu J, Zhang J, et al. Fecal calprotectin concentrations in healthy children aged 1-18 months. PLoS One. 2015;10(3): e0119574 PMID: 25742018

14. Liu JR, Sheng XY, Hu YQ, Yu XG, Westcott JE, Miller LV, et al. Fecal calprotectin levels are higher in rural than in urban Chinese infants and negatively associated with growth. BMC Pediatr. 2012;12:129 PMID: 22917269

15. Ballinger AB, Azooz O, El-Haj T, Poole S, Farthing MJ. Growth failure occurs through a decrease in insulin-like growth factor 1 which is independent of undernutrition in a rat model of colitis. Gut. 2000;46(5):694-700 PMID: 10764714

16. DeBoer MD, Vijayakumar V, Gong M, Fowlkes JL, Smith RM, Ruiz-Perez F, et al. Mice with infectious colitis exhibit linear growth failure and subsequent catch-up growth related to systemic inflammation and IGF-1. Nutr Res. 2017:39:34-42 PMID: 28385287.

17. Morris GP, Beck PL, Herridge MS, Depew WT, Szewczuk MR, Wallace JL. Hapten-induced model of chronic inflammation and ulceration in the rat colon. Gastroenterology. 1989;96(3):795-803 PMID: 2914642.

18. Sawczenko A, Azooz O, Paraszczuk J, Idestrom M, Croft NM, Savage MO, et al. Intestinal inflammation-induced growth retardation acts through IL-6 in rats and depends on the -174 IL-6 G/C polymorphism in children. Proc Nat Acad Sci U S A. 2005;102(37):13260-5 PMID: 16150725.

19. Kalla R, Kennedy NA, Ventham NT, Boyapati RK, Adams AT, Nimmo ER, et al. Serum calprotectin: a novel diagnostic and prognostic marker in inflammatory bowel diseases. Am J Gastroenterol. 2016;111(12):1796-805 PMID 27596694

20. Pepper RJ, Draibe JB, Caplin B, Fervenza FC, Hoffman GS, Kallenberg CG, et al. RAVE-Immune Tolerance Network Research Group. Association of serum calprotectin (s100a8/a9) level with disease relapse in proteinase 3antineutrophil cytoplasmic antibody-associated vasculitis. Arthritis Rheumatol. 2017:69(1):185-93 PMID 27428710.

21. Ehrchen JM, Sunderkötter C, Foell D, Vogl T, Roth J. The endogenous Tolllike receptor 4 agonist S100A8/S100A9 (calprotectin) as innate amplifier of infection, autoimmunity, and cancer. J Leukoc Biol. 2009;86(3):557-66 PMID 19451397.

22. Pruenster M, Vogl T, Roth J, Sperandio M. S100A8/A9: From basic science to clinical application. Pharmacol Ther. 2016:167:120-31 PMID 27492899.

23. Fagerberg UL, Lööf $L$, Merzoug RD, Hansson LO, Finkel Y. Fecal calprotectin levels in healthy children studied with an improved assay. J Pediatr Gastroenterol Nutr. 2003:37(4):468-72 PMID 14508218.

24. Lawrence T. The nuclear factor NF-kappaB pathway in inflammation. Cold Spring Harb Perspect Biol. 2009:1(6):a001651 PMID 20457564.

25. Buzzelli MD, Nagarajan M, Radtka JF, Shumate ML, Navaratnarajah M, Lang $\mathrm{CH}$, et al. Nuclear factor-kappaB mediates the inhibitory effects of tumor necrosis factor-alpha on growth hormone-inducible gene expression in liver. Endocrinology. 2008:149(12):6378-88.

26. Han X, Benight N, Osuntokun B, Loesch K, Frank SJ, Denson LA. Tumour necrosis factor alpha blockade induces an anti-inflammatory growth hormone signalling pathway in experimental colitis. Gut. 2007;56(1):73-81.

27. Eivindson M, Grønbaek H, Flyvbjerg A, Frystyk J, Zimmermann-Nielsen E, Dahlerup JF. The insulin-like growth factor (IGF)-system in active ulcerative colitis and Crohn's disease: relations to disease activity and corticosteroid treatment. Growth Horm IGF Res. 2007:17(1):33-40 PMID 17126585.

28. Street ME, de' Angelis G, Camacho-Hübner C, Giovannelli G, Ziveri MA, Bacchini PL, et al. Relationships between serum IGF-1, IGFBP-2, interleukin1 beta and interleukin-6 in inflammatory bowel disease. Horm Res. 2004; 61(4):159-64 PMID 14691340.

29. Lang CH, Nystrom GJ, Frost RA. Tissue-specific regulation of IGF-I and IGFbinding proteins in response to TNF alpha. Growth Horm IGF Res. 2001; 11(4):250-60 PMID 11735242

30. Bannerjee K, Camacho-Hübner C, Babinska K, Dryhurst KM, Edwards R, Savage $\mathrm{MO}$, et al. Anti-inflammatory and growth-stimulating effects precede nutritional restitution during enteral feeding in Crohn disease. J Pediatr Gastroenterol Nutr. 2004:38(3):270-5 PMID 15076624.

31. Wong SC, Smyth A, McNeill E, Galloway PJ, Hassan K, McGrogan P, et al. The growth hormone insulin-like growth factor 1 axis in children and adolescents with inflammatory bowel disease and growth retardation. Clin Endocrinol (Oxf). 2010:73(2):220-8 PMID 20184596.

32. Yang J, Chen Q, Tian S, Song S, Liu F, Wang Q, et al. The role of 1,25dyhydroxyvitamin D3 in mouse liver ischemia reperfusion injury: regulation of autophagy through activation of MEK/ERK signaling and PTEN/PI3K/Akt/ mTORC1 signaling. Am J Transl Res. 2015;7(12):2630-45 PMID 26885262

33. Harris J. Autophagy and cytokines. Cytokine. 2011;56(2):140-4 PMID 21889357

\section{Publisher's Note}

Springer Nature remains neutral with regard to jurisdictional claims in published maps and institutional affiliations. 